\title{
SIMPLE CONFORMALLY RECURRENT SPACE-TIMES ARE CONFORMALLY RECURRENT PP-WAVES
}

\author{
CARLO ALBERTO MANTICA AND LUCA GUIDO MOLINARI
}

\begin{abstract}
We show that in dimension $n \geq 4$ the class of simple conformally recurrent space-times coincides with the class of conformally recurrent pp-waves.
\end{abstract}

\section{INTRODUCTION}

Riemannian manifolds with recurrent Weyl tensor (conformally recurrent manifolds) were introduced by Adati and Miyazawa [1] and subsequently extended to pseudo-Riemannian manifolds by Derdziński [7], Roter [31, 32, 33, 34, Suh and Kwon [38, and to Lorentzian manifolds (spacetimes) by Mc Lenaghan et al. [24, 25], Hall [15, 16], De and Mantica [5]. In a recent study on conformally recurrent pseudo-Riemannian and Lorentzian manifolds in dimension $n \geq 5$, the authors showed that the Ricci tensor has at most two different eigenvalues, with restrictions on the metric structure, and gave an explicit representation of the Weyl tensor as a Kulkarni-Nomizu product [21, 22].

A pseudo-Riemannian manifold of dimension $n \geq 4$ is "conformally recurrent" if the Weyl curvature tenson 1 is everywhere non-zero and satisfies the condition

$$
\nabla_{i} C_{j k l m}=\alpha_{i} C_{j k l m}
$$

where $\alpha_{i}$ is a non-zero 1 -form named recurrence vector.

The natural question arises whether the recurrent vector may be locally cancelled by a conformal transformation. This "simple" case was investigated by Roter [31, 34].

Date: Nov 2016.

2010 Mathematics Subject Classification. Primary 53B30; Secondary 53C80.

Key words and phrases. conformally recurrent pseudo-Riemannian manifold, simple conformally recurrent pseudo Riemannian manifold, pp-wave space-time.

${ }^{1}$ The local components of the Weyl tensor are 29.

$$
C_{j k l}{ }^{m}=R_{j k l}{ }^{m}+\frac{1}{n-2}\left(\delta_{j}^{m} R_{k l}-\delta_{k}^{m} R_{j l}+g_{k l} R_{j}^{m}-g_{j l} R_{k}{ }^{m}\right)-R \frac{\delta_{j}^{m} g_{k l}-\delta_{k}^{m} g_{j l}}{(n-1)(n-2)}
$$

with Ricci tensor $R_{i j}=-R_{k i j}{ }^{k}$ and curvature scalar $R=R_{k}^{k}$. 
Definition 1.1 (Roter). A conformally recurrent manifold $(\mathscr{M}, g)$ is "simple" if the metric is locally conformal to a conformally symmetric metric, i.e. at each point $x \in \mathscr{M}$ there exist a neighbourhood $U_{x}$ and a scalar function $\sigma$ on $U_{x}$ such that $g_{i j}^{\prime}=g_{i j} e^{2 \sigma}$ and $\nabla_{i}^{\prime} C_{j k l m}^{\prime}=0$ on $U_{x}$.

The main result of this work is the statement that such spaces, with a Lorentzian metric, coincide with (conformally recurrent) pp-wave Lorentzian space-times.

pp-waves are important in general relativity (see for example [19] and references therein). They were studied in $n>4$ by Schimming [36], and appear in Kaluza-Klein theories and in string theory. In the literature (see for example [27]), pp-wave space-times are identified with Brinkmann-waves, i.e. space-times equipped with a null covariantly constant vector field, $X^{i} X_{i}=$ $0, \nabla_{j} X_{k}=0$ [4]. Here we conform to the more restrictive definition used in [36, 19, 17, 18]:

Definition 1.2. A pp-wave is a Brinkmann-wave whose Riemann tensor satisfies the condition $R_{j k}^{p q} R_{p q l m}=0$.

The layout of the paper is the following. In section 2 we present preliminary material about simple conformally recurrent pseudo-Riemannian manifolds and pp-wave space-times. In section 3 we prove that in dimension $n \geq 4$ a conformally recurrent pp-wave space-time is a simple conformally recurrent Lorentzian manifold, and establish the converse implication. We obtain new curvature properties of conformally recurrent pp-waves, such as $\nabla^{m} \nabla_{m} R_{i j}=0$. In Section 4 we show that conformally recurrent ppwave space-times are solutions to the extended theory of gravity with pure radiation source.

Throughout the paper, the manifolds are assumed to be connected, Hausdorff, with non-degenerate metric of arbitrary signature, i.e. $n$-dimensional pseudo-Riemannian manifolds. Some results are restricted to Lorentzian manifolds (space-times) i.e. to metrics of signature $n-2$.

\section{Preliminary Results}

In this section we present preliminary material about conformally recurrent pseudo-Riemannian manifolds, simple conformally recurrent manifolds, and pp-wave space-times.

A vector field belongs to the Olszak distribution [26] if

$$
X_{i} C_{j k l m}+X_{j} C_{k i l m}+X_{k} C_{i j l m}=0 .
$$


The condition was extensively studied in the geometric literature on pseudoRiemannian manifolds (see for example [6, 9, 10, 11]).

A contraction of (2.1) with $g^{i m}$ gives $X^{m} C_{j k l m}=0$. Then, a contraction with $X^{i}$ gives $X^{i} X_{i} C_{j k l m}=0$. If the Weyl tensor is non-zero, the Olszak distribution consists of null vectors, $X^{i} X_{i}=0$.

Lemma 2.1. On a n-dimensional Lorentzian manifold with everywhere non-zero Weyl curvature, the Olszak distribution is one-dimensional.

Proof. Suppose that, besides $X_{i}$, there exists another covector $Y_{i}$ satisfying (2.1). Then also $X_{i}+Y_{i}$ belongs to the Olszak distribution, $X^{i} X_{i}=0$, $Y^{i} Y_{i}=0$ and $X_{i} Y^{i}=0$. On a Lorentzian manifold, two null orthogonal vectors must be collinear, $Y_{i}=\mu X_{i}$ [35].

The following general identity holds for the Weyl tensor on a pseudoRiemannian manifold (eq. 3.8 in [1]):

$$
\begin{aligned}
& \nabla_{i} C_{j k l}{ }^{m}+\nabla_{j} C_{k i l}{ }^{m}+\nabla_{k} C_{i j l}{ }^{m}=\frac{1}{n-3} \nabla_{p}\left[\delta_{j}^{m} C_{k i l}^{p}\right. \\
& \left.+\delta_{k}^{m} C_{i j l}^{p}+\delta_{i}^{m} C_{j k l}^{p}+g_{k l} C_{j i}{ }^{m p}+g_{i l} C_{k j}{ }^{m p}+g_{j l} C_{i k}{ }^{m p}\right]
\end{aligned}
$$

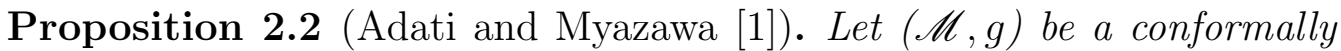
recurrent pseudo-Riemannian manifold of dimension $n \geq 4$. Then:

1) $\nabla_{m} C_{j k l}{ }^{m}=0$ if and only if $\alpha_{i}$ belongs to the Olszak distribution;

2) If $\nabla_{m} C_{j k l}{ }^{m}=0$, then the recurrent covector is null, $\alpha^{i} \alpha_{i}=0$.

Proof. Obviously $\nabla_{m} C_{j k l}{ }^{m}=0$ if and only if $\alpha_{m} C_{j k l}{ }^{m}=0$. The vanishing of the right hand side of (2.2) and recurrence imply that $\alpha_{i}$ belongs to the Olszak distribution. Conversely, we have $\alpha^{i} \alpha_{i} C_{j k l m}=0$, i.e. either $\alpha^{i} \alpha_{i}=0$ or $C_{j k l m}=0$.

Theorem 2.3 (Roter [31, 34]). A pseudo-Riemannian manifold $\mathscr{M}$ of $d i$ mension $n \geq 4$ is simple conformally recurrent if and only if:

1) $C_{j k l m} \neq 0$ everywhere on $\mathscr{M}$,

2) $\nabla_{i} C_{j k l m}=\alpha_{i} C_{j k l m}$, with recurrence 1 -form $\alpha_{i}$ that is locally a gradient,

3) the Ricci tensor is a Codazzi tensor, $\nabla_{k} R_{j l}=\nabla_{j} R_{k l}$.

The contraction of the Codazzi property with the metric tensor gives $\nabla_{k} R=0$, where $R$ is the scalar curvature. Moreover, it was shown:

Proposition 2.4 (Roter [31]). On a non-locally symmetric simple conformally recurrent manifold, $R=0$.

Proposition 2.5 (Roter [34], lemma 12). If the Ricci tensor of a non-locally symmetric simple conformally recurrent manifold has the form $R_{i j}= \pm d_{i} d_{j}$, 
then the following equations hold:

$$
\begin{aligned}
d_{i} C_{j k l m}+d_{j} C_{k i l m}+d_{k} C_{i j l m} & =0, \\
d_{i} R_{j k l m}+d_{j} R_{k i l m}+d_{k} R_{i j l m} & =0 .
\end{aligned}
$$

Proposition 2.6 (Roter [34, theorem 1). Every simple conformally recurrent Lorentzian manifold is Ricci-recurrent, i.e. there exists a non-zero covector field $\omega_{i}$ such that $\nabla_{k} R_{j l}=\omega_{k} R_{j l}$.

Brinkmann and pp-wave space-times arise in the presence of a null covariantly constant vector field. They are special cases of Lorentzian manifolds with a recurrent null vector field, which have been studied for a long time (see for example [39, 4, 36, 17, 18]). A characterization of the metric through a set of canonical coordinates was obtained by Walker [39]. We recall Proposition 1 of [18]:

Proposition 2.7. Let $(\mathscr{M}, g)$ be a Lorentzian manifold of dimension $n=$ $d+2>2$ with a recurrent null vector field, $X^{k} X_{k}=0, \nabla_{k} X_{j}=p_{k} X_{j}$.

1) This is equivalent to the existence of coordinates $\left(u, x^{1}, \ldots, x^{d}, v\right)$, in which the metric has the local expression

$$
d s^{2}=2 d u d v+H(u, \vec{x}, v) d u^{2}+\sum_{\rho=1}^{d} a_{\rho}(u, \vec{x}) d u d x^{\rho}+\sum_{\mu, \nu=1}^{d} g_{\mu \nu}^{*}(u, \vec{x}) d x^{\mu} d x^{\nu},
$$

where $g_{\mu \nu}^{*}$ and $a_{\rho}$ are independent of the coordinate $v$, and $H$ is a smooth function. We refer to these coordinates as Walker coordinates.

2) $\nabla_{k} X_{j}=0$ if and only if $H$ does not depend on $v$. We refer to these coordinates as Brinkmann coordinates, and to the manifold as a Brinkmannwave (space-time) [4].

A pp-wave is a Brinkmann-wave with some restrictions. Schimming [36] gave the following coordinate characterization (see also [19, 17, 18]):

Proposition 2.8 (Schimming [36]). A Lorentzian manifold of dimension $d+2>2$ is a pp-wave if and only if there exist coordinates $(u, \vec{x}, v)$ in which the metric has the following local expression:

$$
d s^{2}=2 d u d v+H(\vec{x}, u) d u^{2}+\sum_{\rho=1}^{d} d x^{\rho 2}
$$

where $H(\vec{x}, u)$ is a smooth function independent of $v$, usually called the "potential function of the pp-wave". 
The expression of the metric yields the Ricci tensor of a pp-wave:

$$
R_{k l}=\psi(\vec{x}, u) X_{k} X_{l}, \quad \psi(\vec{x}, u)=-\frac{1}{2} \sum_{\rho=1}^{d} \frac{\partial^{2} H}{\partial x^{\rho 2}}
$$

where $X_{k}=\nabla_{k} u$ is a covariantly constant null vector $\left(X^{k} X_{k}=0, \nabla_{j} X_{k}=\right.$ 0) [28]. It follows that the scalar curvature is zero, $R=0$.

Remark 2.9. A metric such that $R_{k l}=\psi X_{k} X_{l}$ with a null recurrent covector is called a pure radiation metric with parallel rays, or aligned pure radiation metric [20].

Proposition 2.10 (Schimming [36], see also [19, 17, 18]). A Lorentzian manifold of dimension $d+2>2$ with covariantly constant null vector field (i.e. a Brinkmann wave) is a pp-wave if and only if one of the following conditions is satisfied:

$$
\begin{aligned}
& X_{i} R_{j k l m}+X_{j} R_{k i l m}+X_{k} R_{i j l m}=0 ; \\
& R_{j k l m}=X_{j} X_{m} D_{k l}-X_{j} X_{l} D_{m k}-X_{k} X_{m} D_{j l}+X_{k} X_{l} D_{j m} ; \\
& R^{p}{ }_{j k}^{q} R_{p l m q}=\chi X_{j} X_{k} X_{l} X_{m} ;
\end{aligned}
$$

$D_{i j}$ is a symmetric tensor and $\chi$ is a suitable scalar function.

A contraction recovers the form of the Ricci tensor of a pp-wave, $R_{k l}=$ $\psi X_{k} X_{l}$. Leistner and Nurowski showed that in dimension $d+2=4$ the conditions are equivalent to $R_{j k}^{p q} R_{p q l m}=0$ [19].

Remark 2.11. It is worth noting that for a Ricci tensor of the form (2.6) we have

$$
X_{i} C_{j k l m}+X_{j} C_{k i l m}+X_{k} C_{i j l m}=X_{i} R_{j k l m}+X_{j} R_{k i l m}+X_{k} R_{i j l m} .
$$

$A$ contraction with $g^{i m}$ and the equality $R_{i j}=\psi X_{i} X_{j}$ imply $X^{m} C_{j k l m}=$ $X^{m} R_{j k l m}$.

For a pp-wave, by eq.(2.7), we have $X_{i} C_{j k l m}+X_{j} C_{k i l m}+X_{k} C_{i j l m}=0$, and so $X^{m} C_{j k l m}=X^{m} R_{j k l m}=0$.

\section{Simple CONFORMally RECURRENT LORENTZian MANiFOldS AND PP-WAVES.}

In this section we obtain two important results. First we show, in several steps, that a conformally recurrent pp-wave space-time is a simple conformally recurrent space-time. Next, we show that a simple conformally recurrent space-time is a pp-wave space-time. Finally, some curvature properties of conformally recurrent pp-wave space-times are presented, as well as some 
technical results.

Lemma 3.1. On a pp-wave space-time of dimension $n \geq 4$, the Ricci tensor: 1) is recurrent with a closed recurrence 1-form, 2) it satisfies $\left[\nabla_{i}, \nabla_{j}\right] R_{k l}=$ 0, 3) it satisfies

$$
\nabla^{j} \nabla^{m} C_{j k l m}=-\frac{n-3}{n-2} \nabla^{2} R_{k l}
$$

where $\nabla^{2}=\nabla_{k} \nabla^{k}$. In particular, $\nabla^{2} R_{k l}=0$ if and only if $\nabla^{j} \nabla^{m} C_{j k l m}=0$.

Proof. In a coordinate system with (2.5) one has $\nabla_{j} R_{k l}=\left(\nabla_{j} \psi\right) X_{k} X_{l}=$ $\left(\nabla_{j} \log |\psi|\right) R_{k l}$. From this we infer $\left[\nabla_{i}, \nabla_{j}\right] R_{k l}=0$, i.e. a pp-wave spacetime is Ricci semi-symmetric [30].

The divergence of the Weyl tensor has the general expression

$$
\nabla_{m} C_{j k l}{ }^{m}=\frac{n-3}{n-2}\left(\nabla_{k} R_{j l}-\nabla_{j} R_{k l}\right)+\frac{n-3}{2(n-1)(n-2)}\left(g_{k l} \nabla_{j} R-g_{j l} \nabla_{k} R\right)
$$

A further covariant divergence gives

$$
\nabla^{j} \nabla^{m} C_{j k l m}=-\frac{n-3}{n-2}\left[\nabla^{2} R_{k l}-\frac{g_{k l} \nabla^{2} R}{2(n-1)}-\left[\nabla_{j}, \nabla_{k}\right] R_{l}{ }^{j}\right]+\frac{n-3}{2(n-1)} \nabla_{k} \nabla_{l} R .
$$

Ricci semi-symmetry and the property $R=0$ give (3.1).

Lemma 3.2 (see [21] and [23] theorem 6.1.). A pp-wave space-time of dimension $d+2 \geq 4$ with the metric (2.5) satisfies $\nabla_{m} C_{j k l}{ }^{m}=0$ if and only if $\nabla_{k} \psi=\lambda X_{k}$.

Proof. For a pp-wave space-time we have $\nabla_{j} R_{k l}=\left(\nabla_{j} \psi\right) X_{k} X_{l}$ and $R=0$. Eq.(3.2) becomes:

$$
\nabla_{m} C_{j k l}^{m}=\frac{n-3}{n-2}\left[\left(\nabla_{k} \psi\right) X_{j}-\left(\nabla_{j} \psi\right) X_{k}\right] X_{l} .
$$

It follows that $\nabla_{m} C_{j k l}{ }^{m}=0$ if and only if $\left(\nabla_{k} \psi\right) X_{j}=\left(\nabla_{j} \psi\right) X_{k}$, which is equivalent to the condition $\nabla_{k} \psi=\lambda X_{k}$ for some scalar function.

Remark 3.3. For a pp-wave the condition $\nabla_{m} C_{j k l}^{m}=0$ is equivalent to $\nabla_{k} R_{j l}=\nabla_{j} R_{k l}$, i.e. to the Ricci tensor being a Codazzi tensor. This follows from (3.2) and $R=0$.

Proposition 3.4. Let $(\mathscr{M}, g)$ be a $d+2 \geq 4$ dimensional pp-wave spacetime with the metric (2.5). If $\psi=-\frac{1}{2} \sum_{\rho=1}^{d} \partial^{2} H / \partial x_{\rho}^{2}$ only depends on $u$, then $\nabla^{2} R_{k l}=0$.

Proof. If $\psi=\psi(u)$ we have $\nabla_{k} \psi=\partial_{k} \psi(u)=\psi^{\prime}(u) X_{k}$ and $\nabla_{m} C_{j k l}{ }^{m}=0$ by the previous lemma.

Therefore $\nabla_{k} R_{i j}=\psi^{\prime} X_{k} X_{i} X_{j}$ and $\nabla^{2} R_{i j}=\psi^{\prime \prime}(u) X^{k} X_{k} X_{i} X_{j}=0$. 
Galaev classified the indecomposable conformally recurrent Lorentzian manifolds [12]. He showed that either the manifold is conformally flat, or $\nabla_{i} R_{j k l m}=0$, or it is a pp-wave. Then he found all pp-wave potential functions $H(\vec{x}, u)$ such that the Weyl tensor in the metric (2.5) is recurrent, $\nabla_{i} C_{j k l m}=\alpha_{i} C_{j k l m}$. The potential solves the following system of $d+1$ equations:

$$
\begin{aligned}
& \left(\alpha_{\rho}-\partial_{\rho}\right) \Omega_{\mu \nu}=0 \quad(\rho=1 \ldots d) \\
& \left(\alpha_{u}-\partial_{u}\right) \Omega_{\mu \nu}=0
\end{aligned}
$$

where $\Omega_{\mu \nu}=\partial_{\mu} \partial_{\nu} H-\frac{1}{n} \delta_{\mu \nu} \sum_{\rho} \partial_{\rho}^{2} H(\mu, \nu=1 \ldots d)$.

Let $\Omega^{2}=\sum_{\mu \nu}\left(\Omega_{\mu \nu}\right)^{2}$. In an open set $\mathcal{O} \subset \mathscr{M}$ where $\Omega^{2} \neq 0$ the equations give $\alpha_{\mu}=\frac{1}{2} \partial_{\mu} \log \Omega^{2}$ and $\alpha_{u}=\frac{1}{2} \partial_{u} \log \Omega^{2}$. The potential for which the metric (2.5) is conformally recurrent turns out to be

$$
H(\vec{x}, u)=\sum_{\rho=1}^{d} x_{\rho}^{2}\left[a(u)+F(u) \lambda_{\rho}^{2}\right]
$$

with functions $a(u), F(u)$ and real numbers $\lambda_{1}+\cdots+\lambda_{d}=0$. Correspondingly, $\psi(\vec{x}, u)=-n a(u)$ and, since it only depends on $u$, we conclude that for conformally recurrent pp-waves the divergence of the conformal tensor vanishes:

Proposition 3.5. On a conformally recurrent pp-wave space-time with the metric (2.5), we have $\nabla_{m} C_{j k l}{ }^{m}=0$; moreover there exists a domain $\mathcal{O}$ where the recurrence covector is a gradient.

The recurrence 1-form $\alpha_{i}$ being closed, it follows that $\left[\nabla_{p}, \nabla_{q}\right] C_{j k l m}=0$, i.e. the manifold is Weyl semi-symmetric (see [8]). Moreover, as $\left[\nabla_{i}, \nabla_{j}\right] R_{k l}=$ 0 , the manifold is also semi-symmetric, $\left[\nabla_{p}, \nabla_{q}\right] R_{j k l m}=0$.

Now, from Proposition 3.5, Remark 3.3 and Theorem 2.3, we have:

Theorem 3.6. In $n \geq 4$ any conformally recurrent pp-wave space-time is a simple conformally recurrent Lorentzian manifold.

Some further properties of conformally recurrent pp-wave space-times are collected here:

Proposition 3.7. For a conformally recurrent pp-wave space-time:

1) the recurrence covector $\alpha_{i}$ is null and collinear with the covariantly constant covector $X_{i}=\nabla_{i} u$

2) the recurrence covector is recurrent with closed recurrence 1-form.

Proof. From Propositions 3.5 and 2.2 we get $\alpha_{i} C_{j k l m}+\alpha_{j} C_{k i l m}+\alpha_{k} C_{i j l m}=0$ i.e. the recurrence covector of a conformally recurrent pp-wave space-time 
belongs to the Olszak distribution. In view of Remark 2.11, for a pp-wave space-time also $X_{i}$ belongs to the Olszak distribution; then, by Lemma 2.1. $\alpha_{i}=\mu X_{i}$. Taking the covariant derivative, we see that $\nabla_{j} \alpha_{i}=\left(\nabla_{j} \mu\right) X_{i}=$ $\left(\nabla_{j} \log |\mu|\right) \alpha_{i} \equiv q_{j} \alpha_{i}$ (i.e. the recurrence vector is itself recurrent, with closed recurrence 1 -form).

Proposition 3.8. Let $(\mathscr{M}, g)$ be a $d+2 \geq 4$ dimensional pp-wave spacetime with the metric (2.5). Then there exists a coordinate domain $\mathcal{O}$ in $\mathscr{M}$ where $\nabla^{2} C_{j k l m}=0$ and $\nabla^{2} R_{j k l m}=0$.

Proof. From the closedness condition $\nabla_{j} \alpha_{i}=\nabla_{i} \alpha_{j}$ we infer $q_{i} \alpha_{j}=q_{j} \alpha_{i}$ so that $q_{j}=\rho \alpha_{i}$ and the recurrence 1 -form $\alpha_{i}$ satisfies $\nabla_{j} \alpha_{i}=\rho \alpha_{i} \alpha_{j}$ and $\nabla^{i} \alpha_{i}=0$. Thus, since $\alpha^{i} \alpha_{i}=0$, we have $\alpha^{i} \nabla_{i} C_{j k l m}=0$ and consequently $\nabla^{i} \nabla_{i} C_{j k l m}=\nabla^{i}\left(\alpha_{i} C_{j k l m}\right)=\left(\nabla^{i} \alpha_{i}\right) C_{j k l m}+\alpha^{i}\left(\nabla_{i} C_{j k l m}\right)=0$. Moreover we have $\nabla^{i} \nabla_{i} R_{k l}=0$ so that $\nabla^{i} \nabla_{i} R_{j k l m}=0$.

Now we consider a $n$-dimensional Lorentzian simple conformally recurrent manifold and show that it is a conformally recurrent pp-wave spacetime.

Theorem 3.9. Let $(\mathscr{M}, g)$ be a Lorentzian simple conformally recurrent manifold of dimension $n \geq 4$ : then there exists a coordinate domain $\mathcal{O}$ in $\mathscr{M}$ where the manifold is a pp-wave space-time.

Proof. From Theorem 2.3 and Proposition 2.4 we have $\nabla_{m} C_{j k l}{ }^{m}=0$ so that $\alpha^{m} C_{j k l m}=0$ and $\alpha_{i} C_{j k l m}+\alpha_{j} C_{k i l m}+\alpha_{k} C_{i j l m}=0$. Taking the covariant derivative, we get $\left(\nabla_{p} \alpha_{i}\right) C_{j k l m}+\left(\nabla_{p} \alpha_{j}\right) C_{k i l m}+\left(\nabla_{p} \alpha_{k}\right) C_{i j l m}=0$. Since in a Lorentzian manifold the Olszak distribution is one-dimensional (Lemma 2.1), this implies proportionality, $\nabla_{i} \alpha_{j}=p_{i} \alpha_{j}$ for some 1 -form $p_{j}$.

Proposition 2.6 states that a simple conformally recurrent Lorentzian manifold is Ricci recurrent, i.e. $\nabla_{k} R_{j l}=\omega_{k} R_{j l}$ for some 1 -form $\omega_{k}$. Furthermore, by Theorem [2.3, the Ricci tensor is Codazzi, $\nabla_{k} R_{j l}=\nabla_{j} R_{k l}$. Then we have

$$
\omega_{j} R_{k l}=\omega_{k} R_{j l}
$$

Contraction with $g^{j l}$ gives $\omega^{l} R_{k l}=0$ because $R=0$ (theorem 2.3). Thus on multiplying (3.7) by $\omega_{k}$ it follows that $\left(\omega^{k} \omega_{k}\right) R_{j l}=0$, i.e. $\omega_{k}$ is a null vector.

Let $\theta^{k}$ be a vector such that $\theta^{k} \omega_{k}=1$. Eq.(3.7) gives $R_{j l}=\omega_{j} \theta^{k} R_{k l}$ and, by symmetry, $\omega_{j} \theta^{k} R_{k l}=\omega_{l} \theta^{k} R_{k j}$. Thus $\theta^{j} R_{j l}=\omega_{l}\left(\theta^{k} \theta^{j} R_{k j}\right)$ from which we finally get:

$$
R_{i j}=\psi \omega_{i} \omega_{j}
$$


where $\psi=\theta^{m} \theta^{j} R_{m j}$ is a scalar function.

Let $d=\psi /|\psi|$ and $d_{j}=\omega_{j} \sqrt{|\psi|}$. Then $R_{i j}=d d_{i} d_{j}$ with $d^{k} d_{k}=0$. By Proposition 2.5, equations (2.3) and (2.4) are recovered. In this way the vector $d_{j}$ belongs to the one-dimensional Olszak distribution: thus $d_{i}=\epsilon \alpha_{i}$ and the Ricci tensor takes the form $R_{j l}=\phi \alpha_{j} \alpha_{l}$. Furthermore, from

$$
\alpha_{i} R_{j k l m}+\alpha_{j} R_{k i l m}+\alpha_{k} R_{i j l m}=0
$$

Remark 2.11 gives: $\alpha^{m} R_{j k l m}=0$. Taking the covariant derivative of $\nabla_{k} \alpha_{l}=$ $p_{k} \alpha_{l}$ and using skew symmetrisation, we obtain $\alpha^{m} R_{j k l m}=\left(\nabla_{j} p_{k}-\nabla_{k} p_{j}\right) \alpha_{l}=$ 0 . Thus the covector $p_{j}$ is locally a gradient, i.e. there exists a coordinate domain $\mathcal{O}$ of $(\mathscr{M}, g)$ where $p_{j}=\nabla_{j} \eta$. The covector $\bar{\alpha}_{j}=\alpha_{j} e^{-\eta}$ is covariantly constant $\left(\nabla_{k} \bar{\alpha}_{j}=0\right)$. Eq.(3.9) with $\bar{\alpha}_{j}$ is Schimming's condition (2.7) for the manifold to be locally a pp-wave space-time.

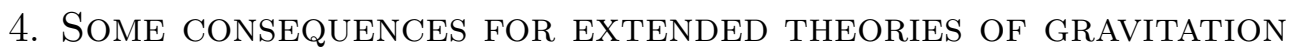

We derive some consequences for extended theories of gravitation in dimensions $n \geq 4$. Since for a pp-wave the scalar curvature is zero, a pp-wave solves the Einstein's field equations (in natural units)

$$
R_{i j}-\frac{1}{2} R g_{i j}=8 \pi T_{i j}
$$

with a pure radiation source, $T_{i j}=\Phi^{2} k_{i} k_{j}$, where $k_{i} k^{i}=0$ (see [37], eq. 5.8).

Generic theories of gravitation modify Einstein's theory at short distances by expressing the action integral with zero source as a power series

$$
\begin{aligned}
I= & \int d^{n} x \sqrt{-g}\left[R-2 \Lambda_{0}+\alpha R^{2}+\beta R_{i j} R^{i j}\right. \\
& \left.+\gamma\left(R_{j k l m} R^{j k l m}-4 R_{j k} R^{j k}+R^{2}\right)+\sum_{p>2} C_{p}(\text { Riem, Ric, } \nabla \text { Riem }, \ldots)\right]
\end{aligned}
$$

where $\alpha, \beta, \gamma, C_{p}$ are parameters provided by some microscopic theory such as string theory. The quadratic part represents "quadratic gravity" (WeylEddington terms), and the terms of higher order are contracted products of the Riemann tensor and its derivatives. Such corrections scale as powers of $\ell_{P} / L$, where $\ell_{P}$ is Planck's length and $L$ is a typical length for the variation of the metric [40], and could be significant in the early evolution of the universe.

The field equations of the full theory are complicated; nevertheless Gürses et al. [14] proved that for pp-wave metrics (2.5) the field equations with 
cosmological constant $\Lambda_{0}=0$ may be written as:

$$
\left[a_{0}+a_{1} \nabla^{2}+a_{2}\left(\nabla^{2}\right)^{2}+\ldots\right] R_{k l}=0
$$

where $a_{i}$ are constants depending on $\alpha, \beta, \gamma, C_{p}$. If $\nabla^{2} R_{k l}=0$ the equation reduces to $a_{0} R_{k l}=0$, with the vacuum solution. In the same paper the authors noted that for pp-waves with $\nabla^{2} R_{k l}=0$ the field equations may include a pure radiation source:

$$
a_{0} R_{k l}=T_{k l}
$$

It is thus interesting to find non-vacuum pp-wave solutions. According to Proposition [3.4, if $\psi=-\frac{1}{2} \sum_{\rho=1}^{d} \partial_{\rho}^{2} H$ depends only on $u$, then $\nabla^{2} R_{k l}=0$.

Proposition 4.1. Let $(\mathscr{M}, g)$ be a $d+2 \geq 4$ dimensional pp-wave space-time with the metric (2.5) : if $\sum_{\rho=1}^{d} \partial_{\rho}^{2} H$ depends only on $u$, then the metric solves the field equations of the generic theory of gravitation with pure radiation source.

We give two examples where $\nabla^{2} R_{k l}=0$ :

1) the pp-wave metric is conformally recurrent, with potential $H$ given by (3.6).

2) the pp-wave metric is two-symmetric i.e. $\nabla_{p} \nabla_{i} R_{j k l m}=0$. This occurs if and only if the potential function of the pp-wave has the form

$$
H(\vec{x}, u)=\sum_{\mu, \nu=1}^{d}\left(u a_{\mu} \delta_{\mu \nu}+b_{\mu \nu}\right) x^{\mu} x^{\nu}
$$

where $0 \leq a_{1} \leq \cdots \leq a_{d}$ and $b_{\mu \nu}=b_{\nu \mu}$ are real numbers (see [2, 3, 13]).

One evaluates $\psi(u)=-\sum_{\mu=1}^{d}\left(u a_{\mu}+b_{\mu \mu}\right)$. Thus for two-symmetric pp-waves space-times the divergence of the conformal tensor vanishes and, as one may directly calculate, $\nabla^{2} R_{k l}=0$.

\section{REFERENCES}

[1] T. Adati and T. Miyazawa, On a Riemannian space with recurrent conformal curvature, Tensor ( N.S.) 18 (1967), 348-354.

[2] D. V. Alekseevsky and A. S. Galaev, Two-symmetric Lorentzian manifolds, J. Geom. Phys. 61 (2011), 2331-2340.

[3] O. F. Blanco, M. Sánchez and J. M. Senovilla, Complete classification of second order symmetric spacetimes, J. Phys.: Conf. ser. 229 (2010), 012021 (5pp).

[4] H. W. Brinkmann, Einstein spaces which are mapped conformally on each other, Math. Ann. 94 (1925), 119-145. 
[5] U.C. De and C. A. Mantica, Conformally recurrent space-times admitting a proper conformal vector field, Commun. Korean Math. Soc. 29 (2) (2014) 319-329.

[6] F. Defever and R. Deszcz, On semi Riemannian manifolds satisfying the condition $R \cdot R=Q(S, R)$, in Geometry and Topology of Submanifolds, III, World Scientific Publ. Singapore. (1991), 108-130.

[7] A. Derdziński, Conformally recurrent indefinite metrics on tori, Tensor (N.S.) 34 (1980), 260-262.

[8] R. Deszcz, Examples of four dimensional Riemannian manifolds satisfying some pseudosymmetry curvature conditions, in Geometry and Topology of Submanifolds II (World Scientific Teaneck, NJ, 1990), pp.134-143.

[9] R. Deszcz and W. Grycak, On certain curvature conditions on Riemannian manifolds, Colloq. Math. 58 (1) (1990), 259-268.

[10] R. Deszcz and M. Hotloś, On a certain extension of the class of semisymmetric manifolds, Publ. de l'Institut Math. (Beograd) (N.S.), tome 63 (77) (1998), 115-130.

[11] R. Deszcz, M. Hotloś, and Z. Sentürk, On the equivalence of the Riccipseudosymmetry and pseudosymmetry, Colloq. Math. 79 (1999), 211221.

[12] A. S. Galaev, Some applications of the Lorentzian holonomy algebras, J. Geom. Symmetry Phys. 26 (2012), 13-31.

[13] S. Gavino-Fernández, The geometry of Lorentzian Ricci solitons, Ph.D. Thesis, Publicaciones del Departamento de Geometria y Topologia, Universidade de Santiago de Compostela, (2012) 105pp.

[14] M. Gürses, T. Ç. Şişman and B. Tekin, AdS-plane wave and pp-wave solutions of generic gravity theories, Phys. Rev. D 90 (2014), 124005.

[15] G. S. Hall, Complex recurrent space-times, J. Phys. A: Math. Nucl. Gen. 7 (1974), L 42-4.

[16] G. S. Hall, Recurrence condition in space-time, J. Phys. A: Math. Gen. 10 (1) (1977), 29-42.

[17] T. Leistner, Conformal holonomy of C-spaces, Ricci-flat, and Lorentzian manifolds, Diff. Geom. Appl. 24 (2006), 458-478.

[18] T. Leistner, Screen bundles of Lorentzian manifolds and some generalizations of pp-waves, J. Geom. Phys. 56 (10) (2006), 2117-2134.

[19] T. Leistner, P. Nurowski, Ambient metrics for n-dimensional pp-waves, Commun. Math. Phys. 296 (3) (2010), 881-898. 
[20] T. Leistner and P. Nurowski, Conformal pure radiation with parallel rays, Class. Quantum Grav. 29 (2012), 055007, (15pp).

[21] C. A. Mantica and L. G. Molinari, On conformally recurrent manifolds of dimension greater than 4, Int. J. Geom. Meth. Mod. Phys. 13 (2016), 1650053 (17 pages).

[22] C. A. Mantica and Y. J. Suh, Recurrent conformal 2-forms on pseudoRiemannian manifolds, Int. J. Geom. Meth. Mod. Phys. 11 (6) (2014), 1450056, (29 pp).

[23] C. A. Mantica and Y. J. Suh, Pseudo-Z symmetric space-times with divergence-free Weyl tensor and pp-waves, Int. J. Geom. Meth. Mod. Phys. 13 (2) (2016), 1650015.

[24] R. G. Mc. Lenaghan and J. Leroy, Complex recurrent space-times, Proc. Roy. Soc. London A 327 (1569) (1972), 229-249.

[25] R. G. Mc Lenaghan and H. A. Thompson, Second order recurrent spacetimes in General Relativity, Lett. Nuovo Cimento 5 (7) (1972) 563-564.

[26] Z. Olszak, On conformally recurrent manifolds, I: Special distributions, Zesz. Nauk. Politech. Śl., Mat.-Fiz. 68 (1993), 213-225.

[27] M. Ortaggio,V. Pravda and A. Pravdova, Algebraic classification of higher dimensional spacetimes based on null alignment, Class. Quantum Grav. 30 (2013), 013001 (57pp).

[28] J. Podolský, and M. Z̆ofka, General Kundt spacetimes in higher dimensions, Class. Quantum Grav. 26 (10) (2009) 105008.

[29] M. M. Postnikov, Geometry VI , Riemannian geometry, Encyclopaedia of Mathematical Sciences, Vol. 91, 2001, Springer-Verlag, Berlin.

[30] W. Roter, Some remarks on second order recurrent spaces, Bull. Acad. Polon. Sci. 12 (1964) 207-211.

[31] W. Roter, On a class of conformally recurrent manifolds, Tensor (N.S.) 39 (1982), 207-217.

[32] W. Roter, On conformally recurrent Ricci-recurrent manifolds, Colloq. Math. 46 (1982), 45-57.

[33] W. Roter, On conformally related conformally recurrent metrics. I Some general results, Colloq. Math. 47 (1982), 315-327.

[34] W. Roter, On the existence of certain conformally recurrent metrics, Colloq. Math. 51 (1987), 39-46.

[35] J. Santos, M. J. Rebouças and A. F. F. Teixeira, Segre types of symmetric two-tensors in n-dimensional space times, Gen. Rel. Grav. 27 (9) (1995), 989-999. 
SIMPLE CONFORMALLY RECURRENT SPACE-TIMES ARE PP-WAVES

[36] R. Schimming, Riemannsche Räaume mit ebenfrontiger und mit ebener Symmetrie, Mathematische Nachrichten 59 (1974), 128-162.

[37] H. Stephani, D. Kramer, M. MacCallum, C. Hoenselaers and E. Hertl, Exact Solutions of Einstein's Field Equations, Cambridge University Press, 2nd ed. (2003).

[38] Y. J. Suh and J-H. Kwon, Conformally recurrent semi-Riemannian manifolds, Rocky Mountain J. of Math. 35 (1) (2005), 285-307.

[39] A. G. Walker, On parallel fields of partially null vector spaces, Quart. J. of Math. 20 (1949), 135-145.

[40] A. Zee, Einstein gravity in a nutshell, Princeton University Press (2013).

I.I.S. Lagrange, Via L. Modignani 65, 20161, Milano, Italy

E-mail address: carloalberto.mantica@libero.it

Physics Department, Università degli Studi di Milano, and I.N.F.N. Sez. Milano, Via Celoria 16, 20133 Milano, Italy

E-mail address: luca.molinari@unimi.it 